\title{
Simulation Models in Gastric Cancer Screening: A Systematic Review
}

\author{
Ayako Matsuda ${ }^{1}$, Kumiko Saika ${ }^{2 *}$, Rina Tanaka ${ }^{3}$, Yuri Ito ${ }^{4}$, Keisuke Fukui ${ }^{4}$, \\ Ken-ichi Kamo5
}

\begin{abstract}
Background: Together with such high-quality approaches as randomized controlled trials and large-scale cohort studies, simulation models are often employed to evaluate the effect of cancer screening methods and decide on their appropriateness. This study aimed to evaluate all effects of gastric cancer screening that have been assessed using simulation models, including cost-effectiveness, mortality reduction, and early-stage detection. Methods: We performed a systematic review using PubMed and Web of Science. We evaluated the effect of screening related to cost, such as incremental cost-effectiveness and incremental cost-effectiveness ratios; we also separately assessed effects other than cost, such as quality-adjusted life-years, number of deaths prevented, life-years saved, relative risk of mortality from gastric cancer, life expectancy, and incidence reduction. The methods targeted for evaluation were Helicobacter pylori testing or endoscopy. Results: We identified 19 studies dealing with simulation models in gastric cancer screenings: 14 examined H. pylori screening and 7 focused on endoscopy. Among those studies, two assessed both $\mathrm{H}$. pylori and endoscopy screening. Most of the studies adopted a Markov model, and all the studies evaluated cost-effectiveness. Of the $14 \mathrm{H}$. pylori screening studies, 13 demonstrated cost-effectiveness and 11 also showed good results other than cost-effectiveness, such as extension of life-years and increase in early-stage detection. In three of the five endoscopy studies, the target population was patients; all five studies obtained good results for cost-effectiveness and four observed good results other than for cost-effectiveness. Conclusions: In this study, we showed that the $H$. pylori screening test was cost-effective in terms of simulation model investigations. However, the H. pylori screening test should not ordinarily be recommended since there is insufficient evidence that it reduces gastric cancer mortality. In Japan, simulation modeling should be employed to plan for cancer control, and the appropriate use of simulation models should be examined for future use.
\end{abstract}

Keywords: Simulation model- gastric cancer- screening- systematic review

Asian Pac J Cancer Prev, 19 (12), 3321-3334

\section{Introduction}

Gastric cancer is one of the leading causes of cancer incidence and mortality in Japan (Hori et al., 2015). Early detection is important toward reducing gastric cancer mortality, and mass screening using photofluorography has been implemented in Japan since 1982. The latest Japanese guidelines for gastric cancer screening published in 2014 by government recommends the use of endoscopy; that recommendation is based on scientific evidence, whereby gastric cancer screening by endoscopy could reduce gastric cancer mortality in a similar fashion to photofluorography (Terasawa et al., 2014). With a recommended means of cancer screening, it should be scientifically demonstrable that the screening is able to detect cancer at an early stage and also reduce cancer mortality. However, serum anti-Helicobacter pylori antibody testing and the serum pepsinogen method were not recommended in the evaluating several screening guidelines (Hamashima et al., 2008). Those two methods were introduced to the 2015 gastric cancer screening program among, respectively, $14.8 \%$ and $11.2 \%$ of Japan's local governments (Ministry of Health, Labour and Welfare, 2017). The above gastric cancer screening guidelines do not recommend screening for the presence of $H$. pylori infection: no quality scientific research has demonstrated the effect of such screening on reducing gastric cancer mortality.

In general, randomized control trials (RCTs) are the most valuable method for evaluating health interventions, including cancer screening, prior to their

${ }^{1}$ Department of Hygiene and Public Health, Teikyo University School of Medicine, ${ }^{3}$ Department of Medical Informatics, Hirosaki University Graduate School of Medicine, Hirosaki, Aomori, Tokyo, ${ }^{3}$ Department of Medical Informatics, Hirosaki University Graduate School of Medicine, Hirosaki, Aomori, ${ }^{4}$ Department of Cancer Epidemiology, Cancer Control Center, Osaka International Cancer Institute, Osaka, ${ }^{5}$ Department of Plastic and Reconstructive Surgery, Sapporo Medical University School of Medicine, Chuo-ku, Sapporo, Japan.*For Correspondence: ksaika@ncc.go.jp 
broad population-based implementation. However, evaluating the effect of cancer screening on mortality reduction demands a long follow-up time and large groups of participants; thus, it is considerably difficult to make an evaluation in terms of such categories as sex, age, and risk factors. Accordingly, simulation models are often applied along with RCTs to ensure proper evaluation of the effects of screening (Koleva-Kolarova et al., 2015). For example, in screening for prostate, breast, and colorectal cancer, computer simulation modeling has been used to estimate the years of life lost as a result of those cancers in 50-year-old renal transplant recipients compared with subjects in the general population (Kiberda et al., 2003). In breast cancer screening, some simulation studies have been performed for mammography screening to determine an appropriate age for screening or to estimate cost-effectiveness (Koleva-Kolarova et al., 2015). In screening for gastric cancer using photofluorography, endoscopy, and H. pylori testing, several simulation studies have been undertaken, and a systematic review has reported the cost-effectiveness (Areia et al., 2013).

In the present study, we aimed to evaluate all the effects of gastric cancer screening, including cost-effectiveness, through a systematic review of all the published studies on gastric cancer screening that made an assessment using simulation models.

\section{Materials and Methods}

We performed a systematic review according to the Preferred Reporting Items for Systematic Reviews and Meta-Analysis checklist (Moher et al., 2009).

\section{Eligibility criteria}

Our inclusion criteria were studies of cancer patients in English- or Japanese-language publications and articles that described simulation studies. We excluded articles that were not original studies complete with full text, studies that were not simulation studies, and statistical studies. We hand searched the trials according to those criteria.

\section{Information sources and search strategy}

We conducted our search on July 11, 2016 in PubMed and Web of Science. The search terms were "gastric cancer," "mass screening," "endoscopy," "X-ray," and "simulation model."

\section{Data items and summary of results}

We collected the following data: first author; publication year; country of study; population (number and age of target population [general population or patients]); type of simulation model; use of sensitivity and validation analysis; details of interventions; and sensitivity and specificity of screening and outcomes. We evaluated the effect related to cost, such as incremental cost-effectiveness and incremental cost-effectiveness ratios; we separately assessed the effect other than cost, such as quality-adjusted life-years (QALYs), number of deaths prevented, life-years saved, relative risk of mortality from gastric cancer, life expectancy, and incidence reduction.
We categorized the subjects into two groups according to the target population for the screening methods: general population and patients. The evaluated screening methods in the simulation were the H. pylori test, endoscopy, and both methods. We summarized the two groups of outcomes according to the screening methods.

\section{Results}

\section{Study characteristics}

The process of study selection appears in Figure 1. Our search resulted in 478 articles in PubMed and 2,361 articles in Web of Science. Two authors independently evaluated the titles and abstracts of all the selected articles using the inclusion criteria and excluded all non-relevant articles. Subsequently, we excluded articles that were not in English or Japanese $(\mathrm{n}=38)$, which resulted in 2,621 articles. Eventually, we identified 19 articles (Parsonnet et al., 1996; Harris et al., 1999; Fendrick et al., 1999; Davies et al., 2002; Mason et al., 2002; Roderick et al., 2003; Leivo et al., 2004; Dan et al., 2006; Lee et al., 2007; Xie et al., 2008; Xie et al., 2008; Shin et al., 2009; Xie et al., 2009; Yeh et al., 2009; Chang et al., 2012; Zhou et al., 2013; Yeh et al., 2016; Yeh et al., 2010; Hassan et al., 2010) that concerned simulation models on gastric cancer screenings.

The articles we found appear in Table 1. Among the 19 studies published between 1996 and 2016, eight were from Asia (China, Singapore, South Korea and, Taiwan), four from Europe (United Kingdom and Finland), six from the United States, and one from Canada. In all, 17 studies (Parsonnet et al., 1996; Harris et al., 1999; Fendrick et al., 1999; Davies et al., 2002; Mason et al., 2002; Roderick et al., 2003; Leivo et al., 2004; Dan et al., 2006; Lee et al., 2007; Xie et al., 2008; Xie et al., 2008; Shin et al.,

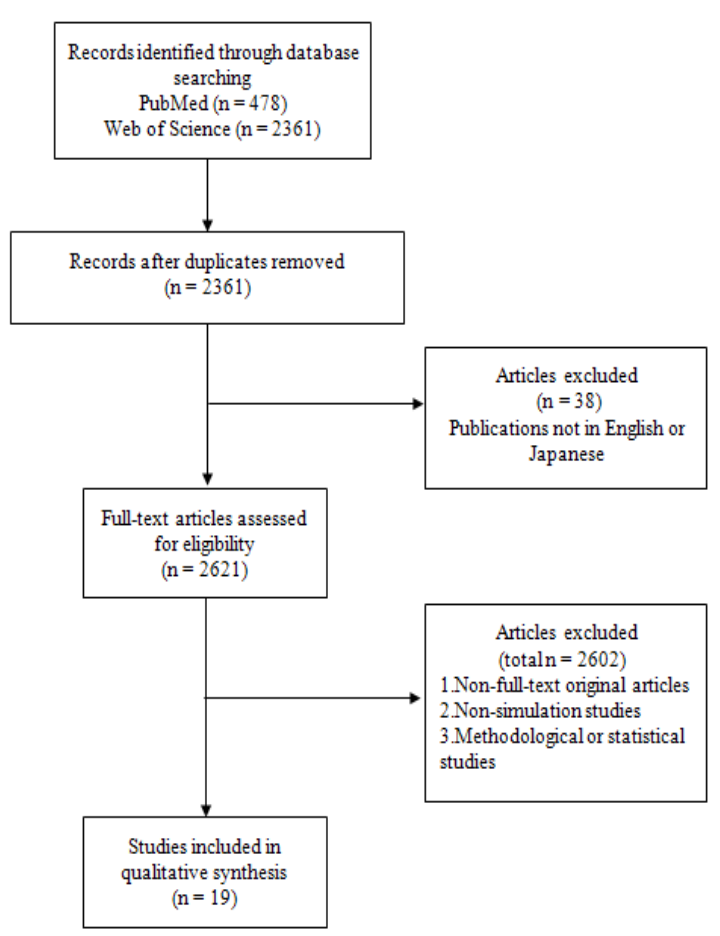

Figure 1. Flow Chart of Article-Selection Process 
DOI:10.31557/APJCP.2018.19.12.3321

Simulation Models in Gastric Cancer Screening: A Systematic Review

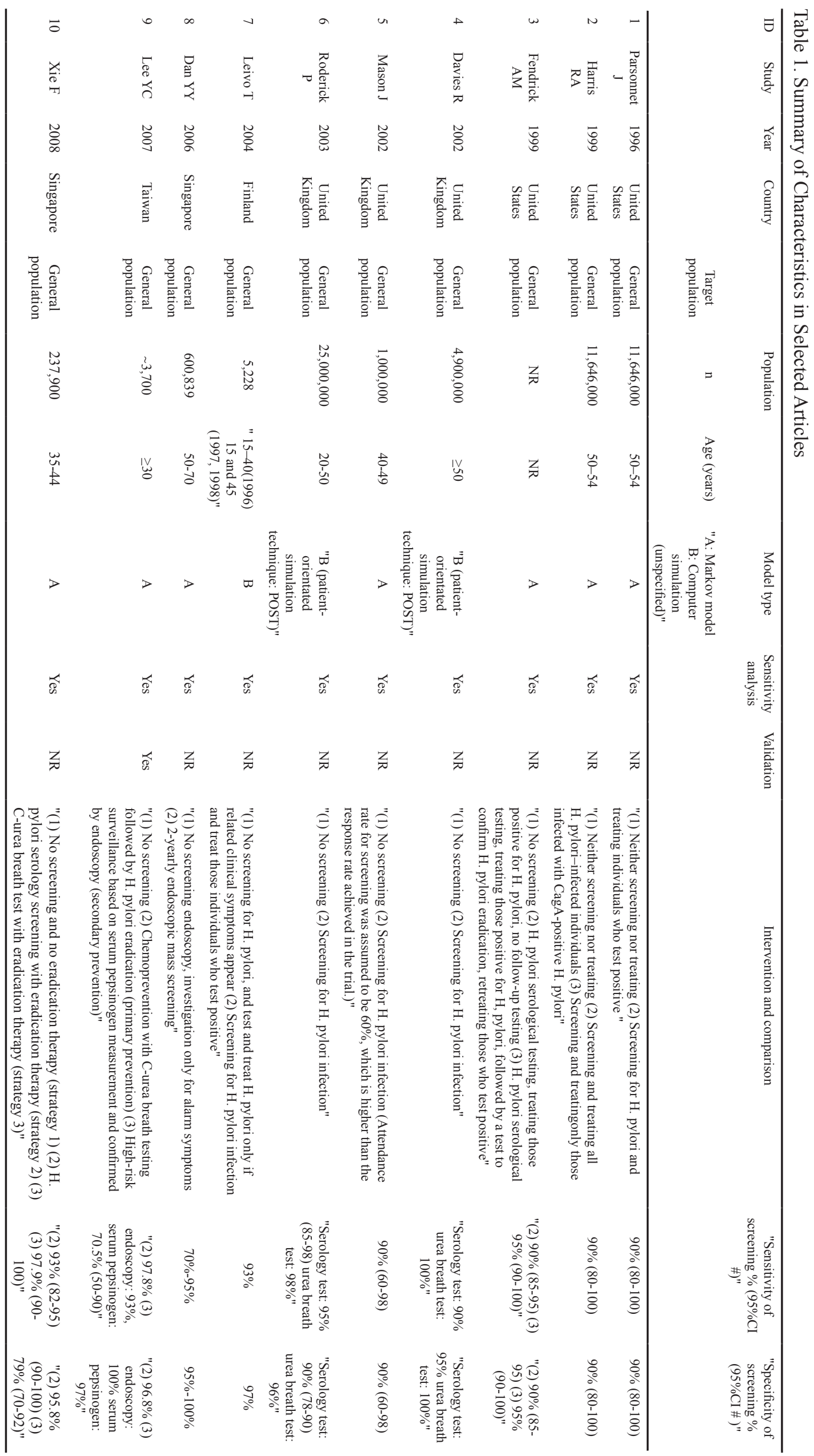




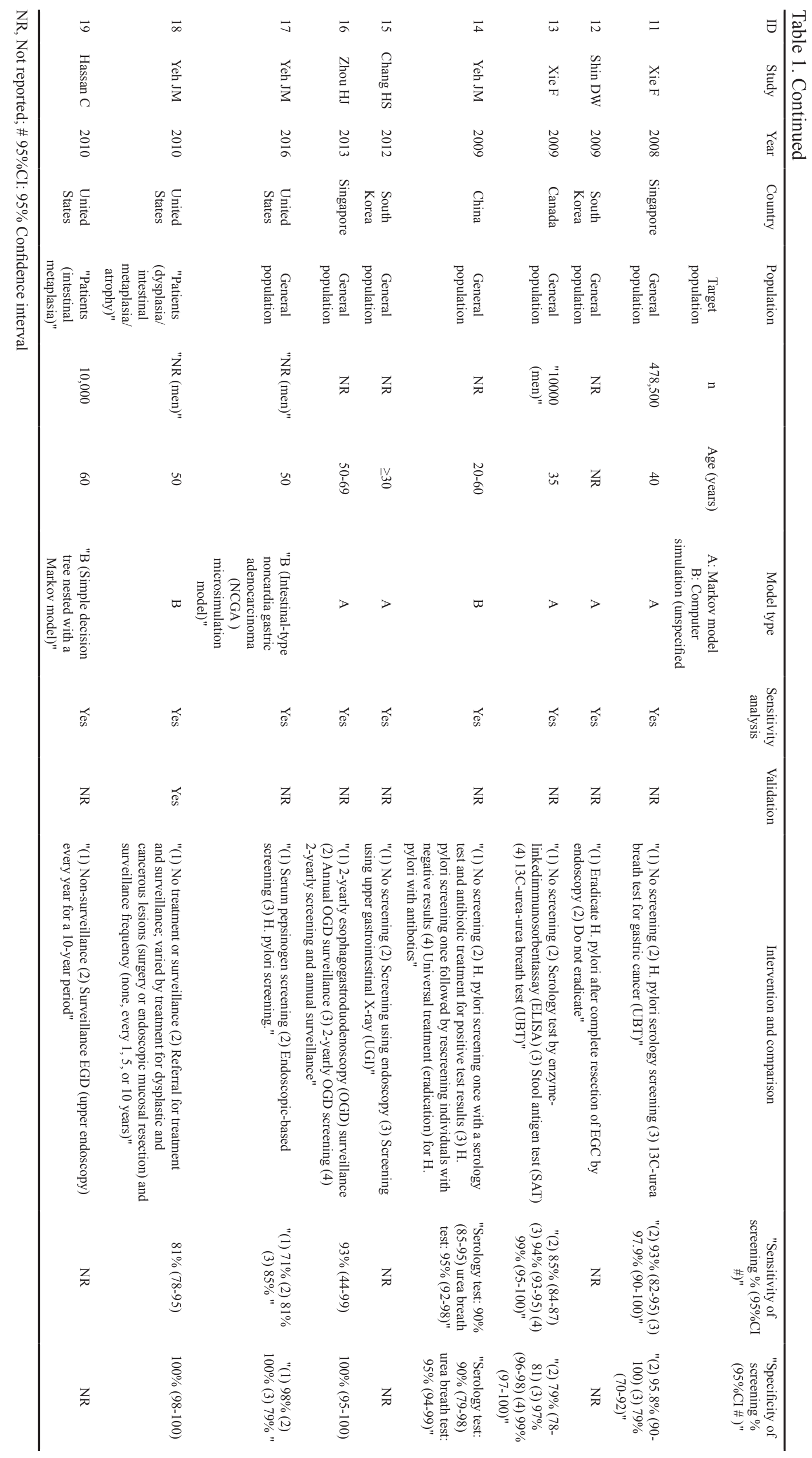


DOI:10.31557/APJCP.2018.19.12.3321

Simulation Models in Gastric Cancer Screening: A Systematic Review

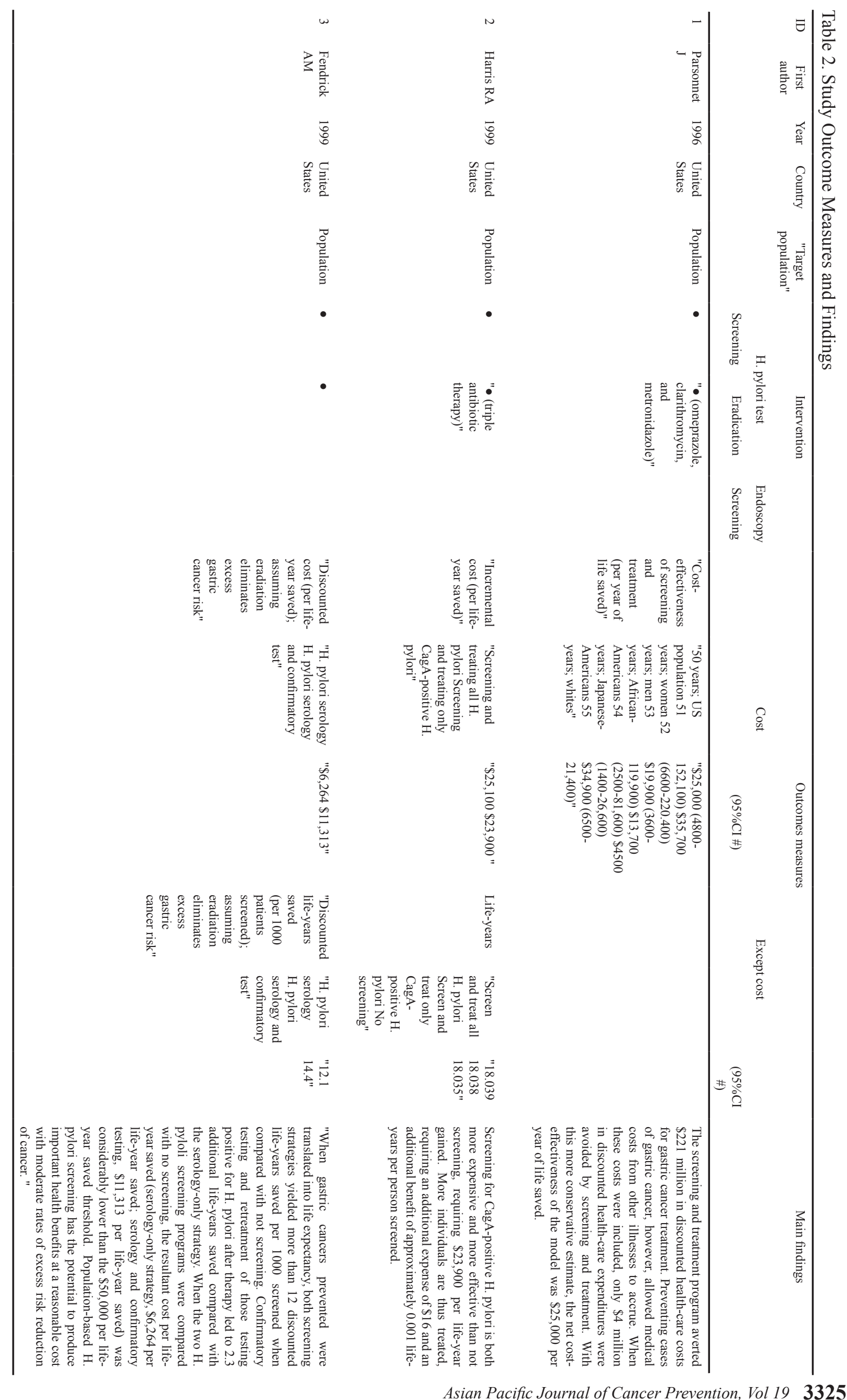




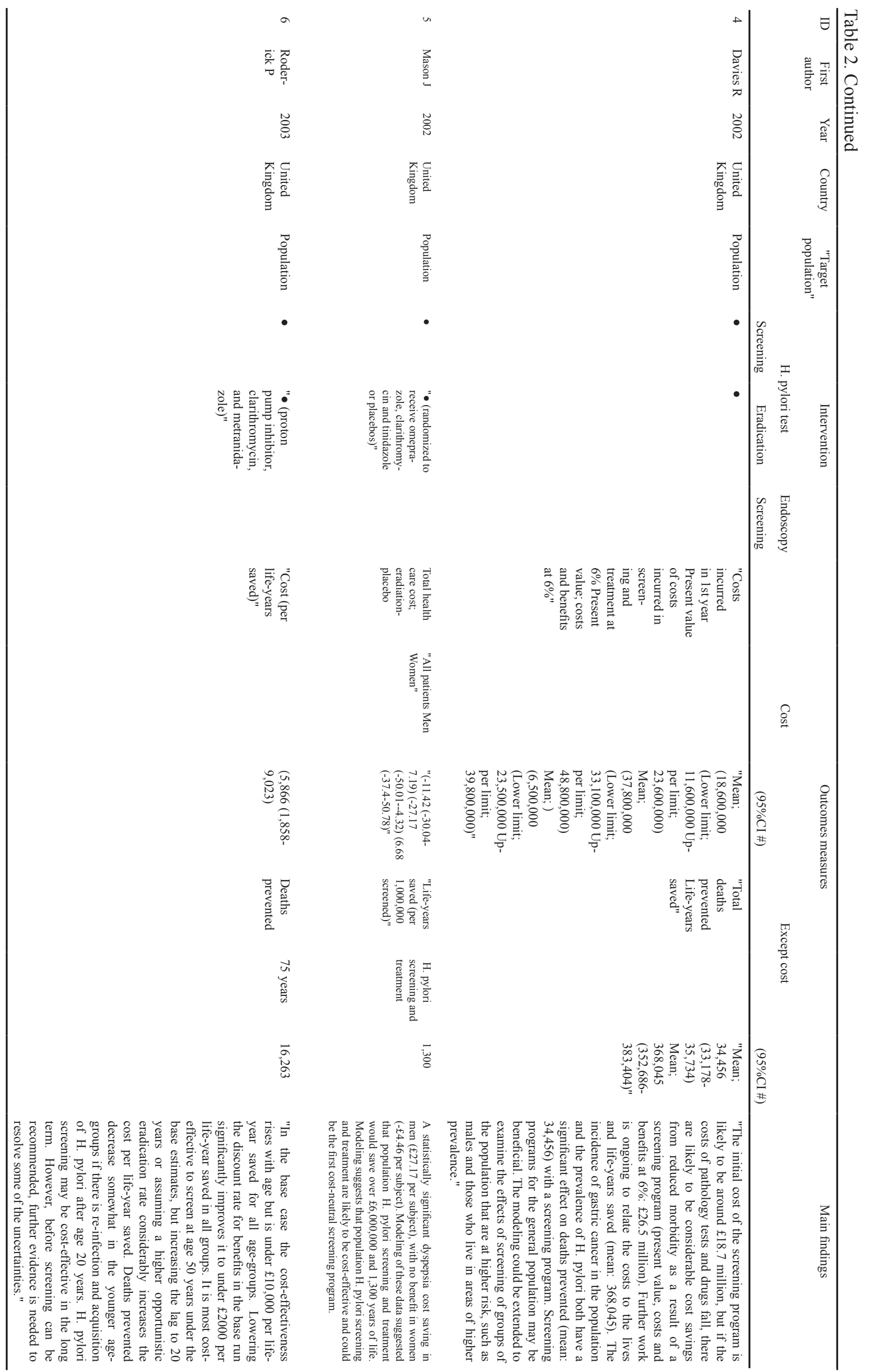

3326 Asian Pacific Journal of Cancer Prevention, Vol 19 
DOI:10.31557/APJCP.2018.19.12.3321

Simulation Models in Gastric Cancer Screening: A Systematic Review
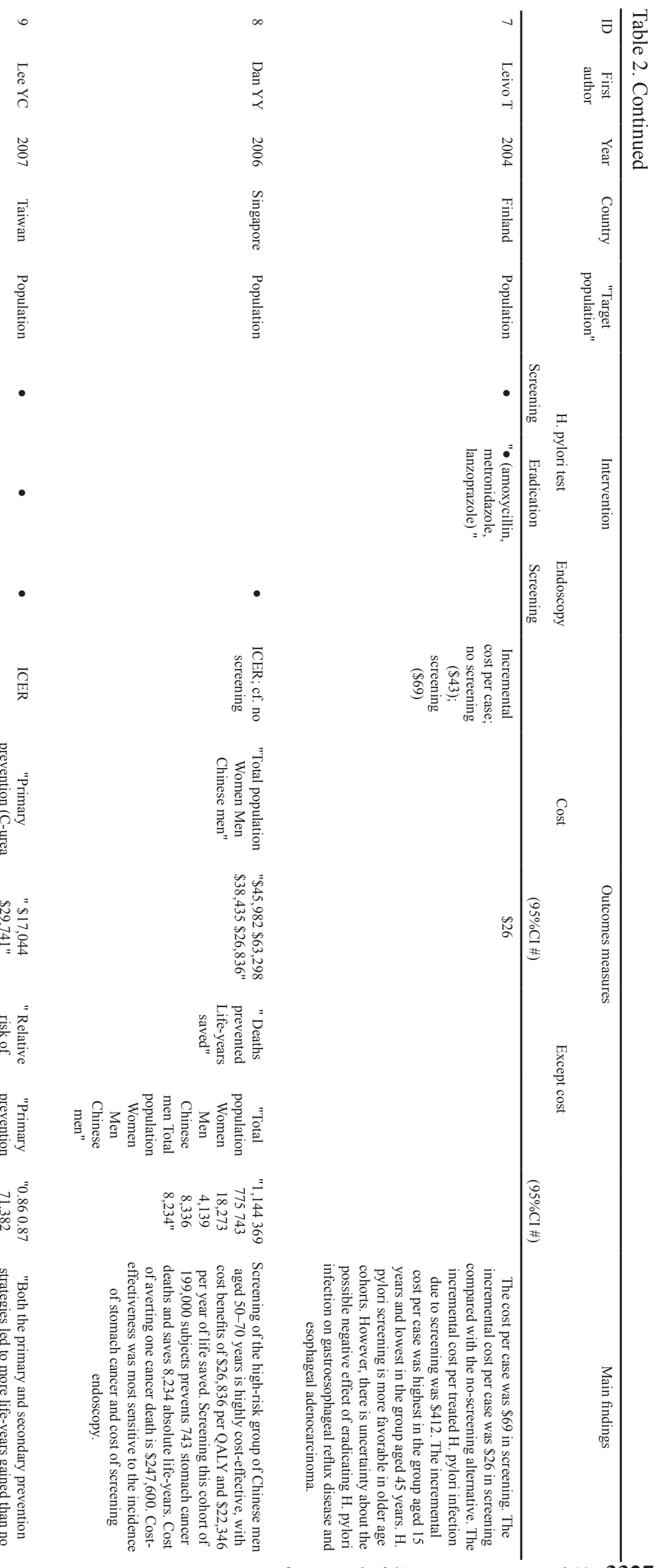
昂管

:

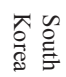

.

苜

.

鄫

尊

.

ज.

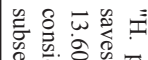

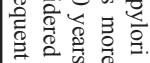

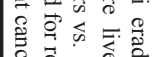

웅.

言它出䛎

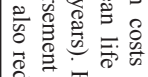

政

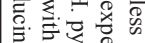

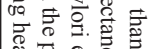

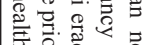

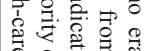

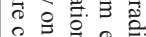

然

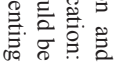

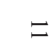

ํ.

莃

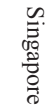

莺

莣

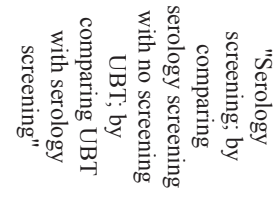

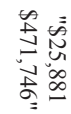

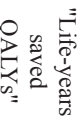

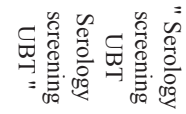

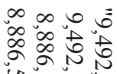

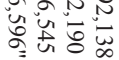

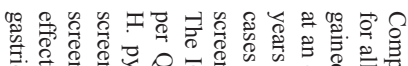

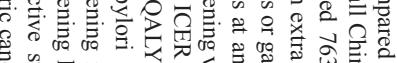

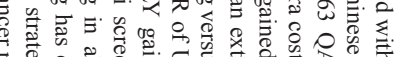

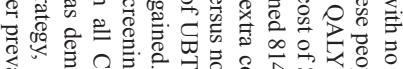

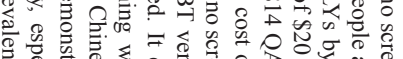

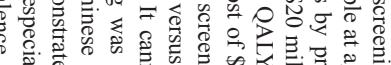

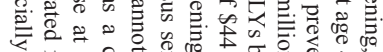

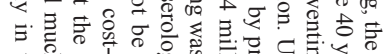

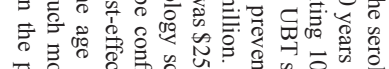

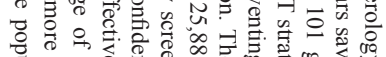

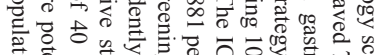

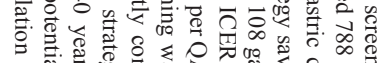

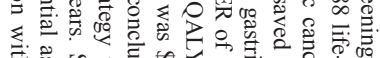
E

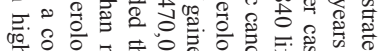

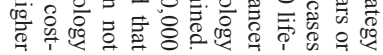

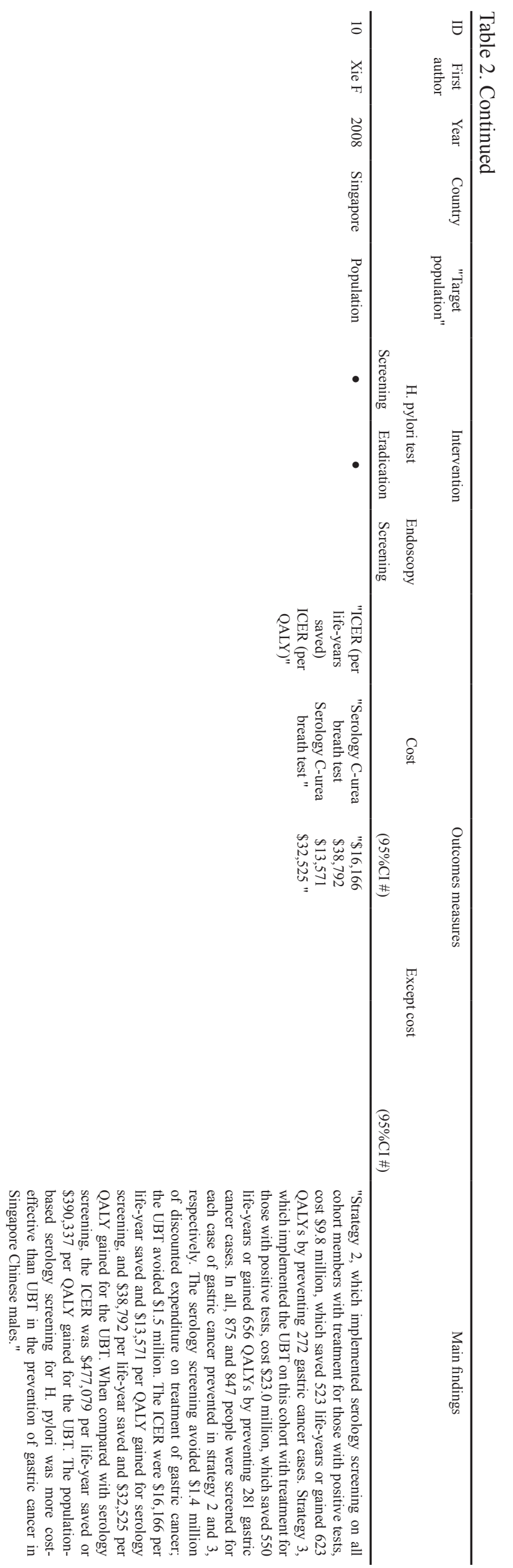


DOI:10.31557/APJCP.2018.19.12.3321

Simulation Models in Gastric Cancer Screening: A Systematic Review

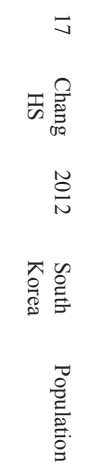

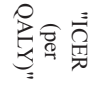

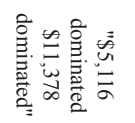

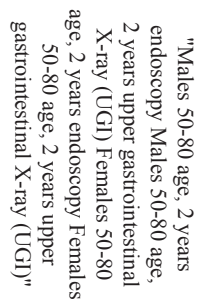

$\mp$

$\xi \underset{\rho}{\sigma}$

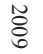

产.

莺

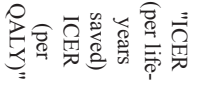

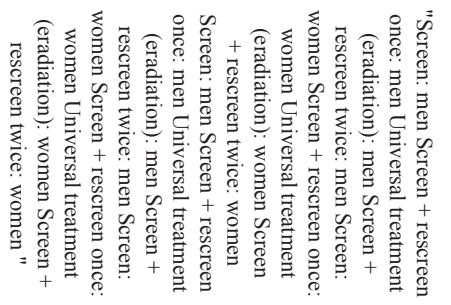

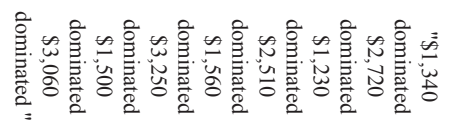

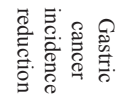

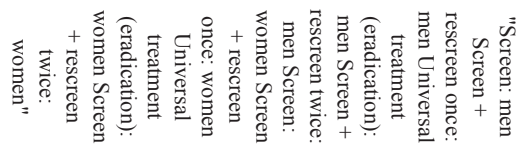

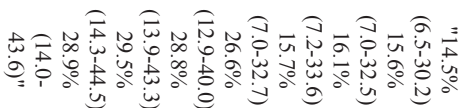

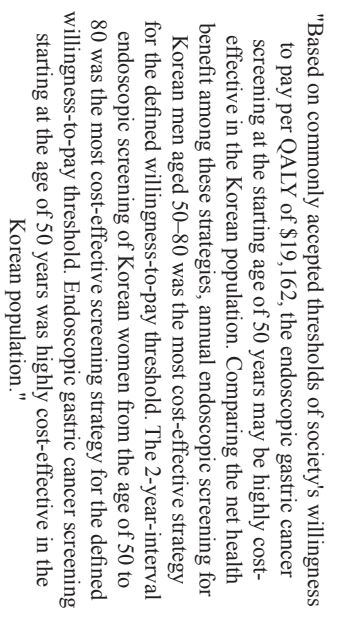

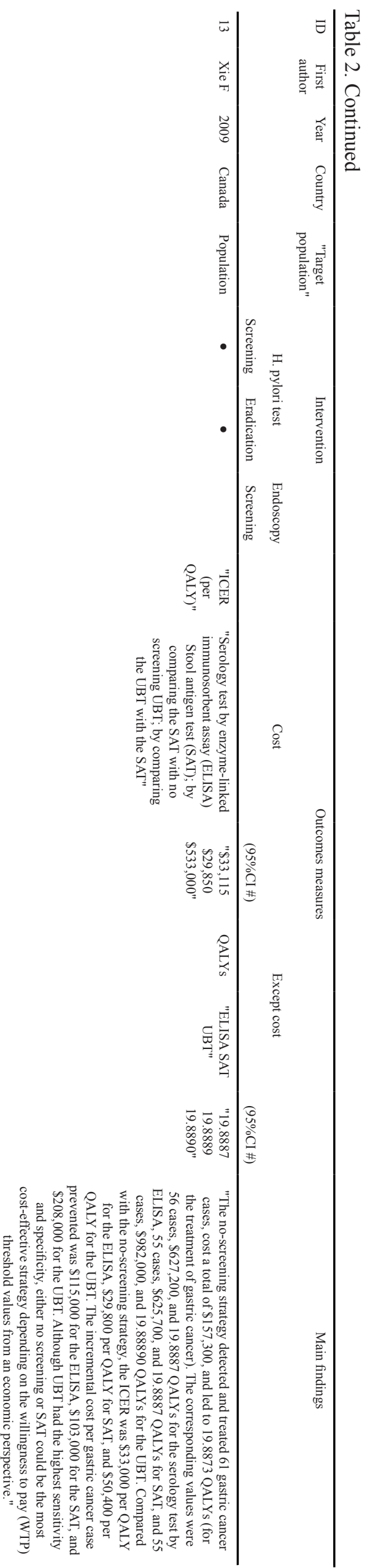



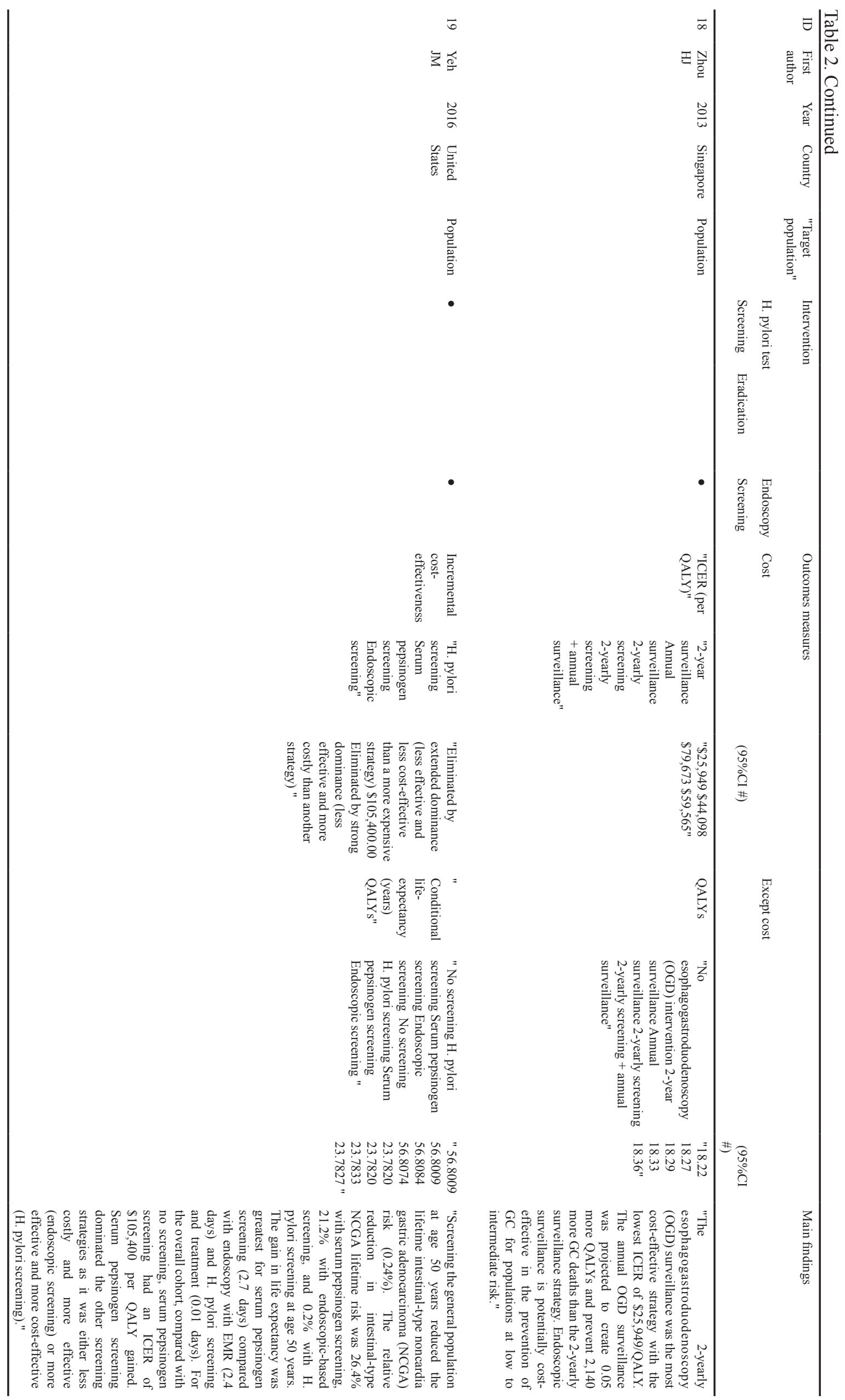


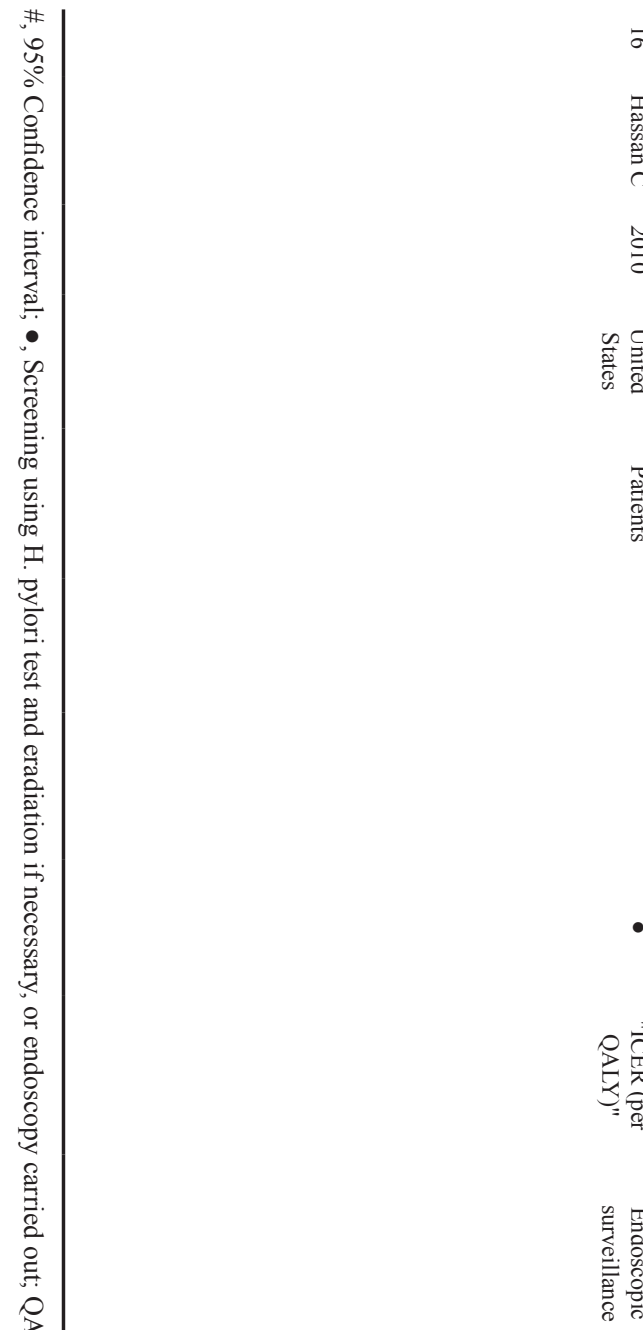

$\bar{\sigma}$

苞

응

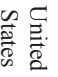

$\stackrel{\overrightarrow{\mathrm{E}}}{\overline{\mathrm{E}}}$

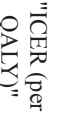

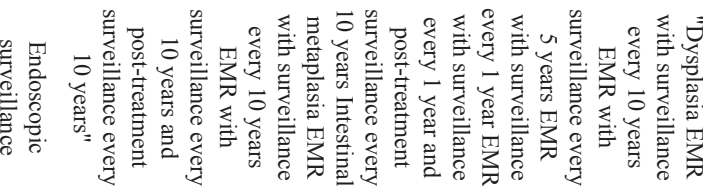

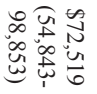

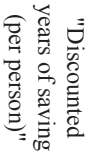

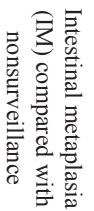

$\dot{\$}$

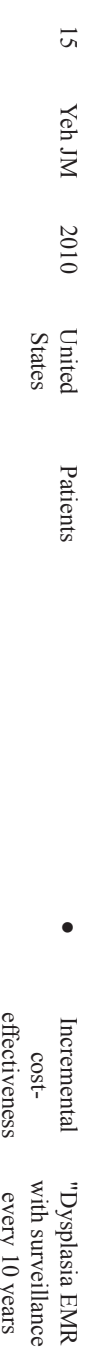

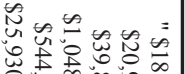

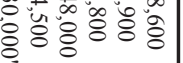

兽

\&

音

๑

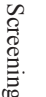

प:

高.

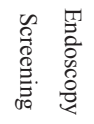

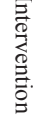

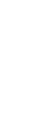

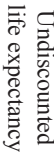

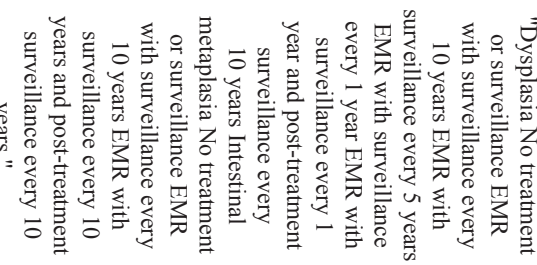

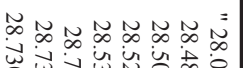

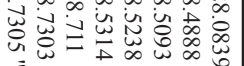

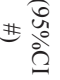

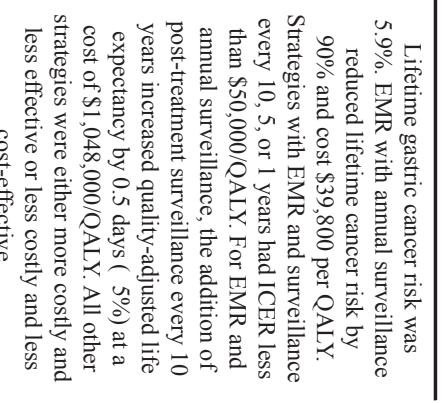

$\forall \mid \overrightarrow{\tilde{\sigma}}$ 
Table 3. Summary of Population Screening Assessment in 17 Studies

\begin{tabular}{lcccc}
\hline Intervention & Outcomes & Effective & Not effective & Total $^{* 4}$ \\
\hline H. pylori & Cost & $14^{*_{1}^{* 2}}$ & $3^{*_{1}^{* 2^{*} 3}}$ & 14 \\
& Except for cost & 11 & 0 & 11 \\
Endoscopy & Cost & $5^{* 2}$ & $1^{*_{2}}$ & 5 \\
& Except for cost & 4 & 0 & 4 \\
\hline
\end{tabular}

${ }^{* 1}$, One study (Mason et al., 2002) showed a sex difference (effective in men, not beneficial in women); ${ }^{* 2}$, One study (Yeh et al., 2016) showed efficacy only in pepsinogen screening; ${ }^{* 3}$, One study (Yeh et al., 2009) showed less cost-effectiveness for the strategy considering eradication, although the strategy that did not consider eradication was cost-effective; ${ }^{* 4}$, Number of studies that evaluated each item.

2009; Xie et al., 2009; Yeh et al.,2009; Chang et al., 2012; Zhou et al., 2013; Yeh et al., 2016) were conducted among healthy populations; two (Yeh et al., 2010; Hassan et al., 2010) were carried out on patients with dysplasia, intestinal metaplasia, or atrophy. Most of the studies adopted a Markov model and performed a sensitivity analysis. With regard to the effect of interventions, the sensitivity and specificity of the $H$. pylori test was set as $81 \%-99 \%$ and $79 \%-100 \%$, respectively; the sensitivity and specificity of endoscopy was set at $70 \%-95 \%$ and $95 \%-100 \%$, respectively.

\section{Assessment of results of main outcomes}

Details of the selected 19 studies appear in Table 2. A summary of the population screening assessment appears in Table 3.

Of all the 19 studies, 14 (Parsonnet et al., 1996; Harris et al., 1999; Fendrick et al., 1999; Davies et al., 2002; Mason et al., 2002; Roderick et al., 2003; Leivo et al., 2004; Lee et al., 2007; Xie et al., 2008; Xie et al., 2008; Shin et al., 2009; Xie et al., 2009; Yeh et al., 2009; Yeh et al., 2016) dealt with $H$. pylori screening. Seven studies (Dan et al., 2006; Lee et al., 2007; Chang et al., 2012; Zhou et al., 2013; Yeh et al., 2016; Yeh et al., 2010; Hassan et al., 2010) covered endoscopy. Two studies (Lee et al., 2007; Yeh et al., 2016) examined both H. pylori and endoscopy screening. All the studies evaluated costeffectiveness, and 15 studies evaluated the outcomes except cost.

Both H. pylori screening and endoscopy screening were found to be cost-effective in all the studies evaluated. However one study (Mason et al., 2002) reported a sex difference, whereby $H$. pylori screening was found to be beneficial for men but not for women. One study (Yeh et al., 2016) determined that serum pepsinogen screening was more cost-effective than $H$. pylori screening, but it was less costly than endoscopic screening. Another study (Yeh et al., 2009) showed that serum pepsinogen screening was less cost-effective in a strategy that considered eradication, although such screening was cost-effective in a strategy that did not consider eradication.

For an evaluation of the effect except cost, among the 11 studies on $H$. pylori screening and four studies on endoscopy screening, all 11 studies on H. pylori (Harris et al., 1999; Fendrick et al., 1999; Davies et al., 2002; Mason et al., 2002; Roderick et al., 2003; Lee et al., 2007;
Xie et al., 2008; Shin et al., 2009; Xie et al., 2009; Yeh et al.,2009; Yeh et al., 2016) and all the studies on endoscopy determined that screening had an effect on the number of deaths prevented, incidence reduction, life-years saved, greater life expectancy, or higher QALYs.

\section{Discussion}

We systematically reviewed published studies on gastric cancer screening that adopted simulation models. In all the selected studies, gastric cancer screening with endoscopy and the $H$. pylori test were cost-effective according to analyses using simulation models. This result is in line with previously reported cost-effectiveness analyses (Areia et al., 2013; Earnshaw et al., 2013). Omidvari et al., (2016) suggested that more research is needed about the efficacy of surveillance to inform more evidence-based cost-effective studies that aim to optimize surveillance programs for gastrointestinal cancers.

Studies on cancer screening using simulation models can provide important information, and the results of the present review are noteworthy. However, it is necessary to evaluate our findings with some caution: the results of simulation studies depend on the quality of the inputted data. That observation is particularly true of studies that do not adopt a good design, such as that of a randomized control study. Assessments based on simulation models are greatly influenced by the inputted data used in those models. For example, among the 14 studies dealing with H. pylori screening, 13 (Parsonnet et al., 1996; Harris et al., 1999; Fendrick et al., 1999; Davies et al., 2002; Mason et al., 2002; Roderick et al., 2003; Leivo et al., 2004; Lee et al., 2007; Xie et al., 2008; Xie et al., 2008; Shin et al., 2009; Xie et al., 2009; Yeh et al., 2016), considered H. pylori eradication as a treatment for individuals with H. pylori infection; the magnitude of eradication varied according to the study. Nine studies (Harris et al., 1999; Davies et al., 2002; Mason et al., 2002; Roderick et al., 2003; Leivo et al., 2004; Xie et al., 2008; Xie et al., 2008; Shin et al., 2009; Xie et al., 2009) determined that eradication of $H$. pylori reduced $30 \%-55 \%$ of the incidence of gastric cancer; those values are similar to ones identified in a meta-analysis (Ford et al., 2014). Several studies found that no gastric cancer occurred among subjects who underwent successful eradication treatment (Parsonnet et al., 1996) or the risk of gastric cancer became the same as among subjects who had never been infected by H. pylori (Fendrick et al., 1999; Lee et al., 2007; Yeh et al., 2009; Yeh et al., 2016).

Thus, simulation analysis for cancer screening strategy should basically not be conducted unless the effect has been demonstrated by means of strong evidence. The US Preventive Services Task Force is developing evidenced-based recommendations about preventive care using models for a preventive service that depend on the service under consideration, state of existing empirical evidence, suitability of models for specific purposes, and available resources (Owens et al., 2016). Therefore the use of modeling studies to develop recommendations should be regarded as supplemental measures. In the Japanese 
guidelines for gastric cancer screening, simulation studies were not considered because the recommendation of a new screening method should be based on strong scientific evidence obtained through highly reliable means, such as randomized control trials and large-scale cohort studies.

In the present study, using simulation model studies we showed that the $H$. pylori screening test was cost-effective. However, that screening test should not ordinarily be recommended because there is a lack of sufficient evidence for gastric cancer screening with $H$. pylori testing being able to reduce gastric cancer mortality, and, therefore, no guidelines in the world recommend its use. Model-based evaluations have been used in health policy discussions and recommendations in such places as the United States and Canada. Simulation models can be used to identify appropriate age-ranges and intervals between screening tests; they cannot be employed to evaluate the effect on main outcomes, such as mortality reduction (Van et al., 1995).

In conclusion, when assessing cancer screening through the appropriate use of simulation models, the results should be beneficial to research and policy decisions. Chang et al., (2012) used Japanese and Korea data in a simulation model. In Japan, it is necessary to employ simulation modeling when planning for cancer control while sufficiently addressing the appropriate future use of simulation models.

\section{Acknowledgments}

This work was supported by Health and Labor Sciences Research Grants and by the Ministry of Education, Culture, Sports, Science and Technology in Japan Grant-in-Aid for Scientific Research Grant B.

We thank the Edanz Group (www.edanzediting.com/ ac) for editing a draft of this manuscript.

\section{References}

Areia M, Carvalho R, Cadime AT, et al (2013). Screening for gastric cancer and surveillance of premalignant lesions: a systematic review of cost effectiveness studies. Helicobacter, 18, 325-37.

Chang HS, Park EC, Chung W, et al (2012). Comparing endoscopy and upper gastrointestinal X-ray for gastric cancer screening in South Korea: a cost-utility analysis. Asian Pac J Cancer Prev, 13, 2721-8.

Dan YY, So JB, Yeoh KG (2006). Endoscopic screening for gastric cancer. Clin Gastroenterol Hepatol, 4, 709-16.

Davies R, Crabbe D, Roderick P, et al (2002). A simulation to evaluate screening for Helicobacter pylori infection in the prevention of peptic ulcers and gastric cancers. Health Care Manag Sci, 5, 249-58.

Earnshaw SR, Brogan AP, McDade CL (2013). Model-based cost effectiveness analyses for prostate cancer chemoprevention:A review and summary of challenges. Pharmaco Economics, 31, 289-304.

Fendrick AM, Chernew ME, Hirth RA, et al (1999). Clinical and economic effects of population-based Helicobacter pylori screening to prevent gastric cancer. Arch Intern Med, 159, 142-8.

Ford AC, Forman D, Hunt RH, et al (2014). Helicobacter pylori eradication therapy to prevent gastric cancer in healthy asymptomatic infected individuals: systematic review and meta-analysis of randomised controlled trials. $B M J, \mathbf{3 4 8}$, g3174.

Hamashima C, Shibuya D, Yamazaki H, et al (2008). The Japanese guidelines for gastric cancer screening. Jpn J Clin Oncol, 38, 259-67.

Harris RA, Owens DK, Witherell H, et al (1999). Helicobacter pylori and gastriccancer: what are the benefits of screening only for the CagA phenotype of H. pylori?. Helicobacter, 4, 69-76.

Hassan C, Zullo A, Di Giulio E, et al (2010). Cost effectiveness of endoscopic surveillance for gastric intestinal metaplasia. Helicobacter, 15, 221-6.

Hori M, Matsuda T, Shibata A, et al (2015). Cancer incidence and incidence rates in Japan in 2009: a study of 32 population-based cancer registries for the Monitoring of Cancer Incidence in Japan (MCIJ) project. Jpn J Clin Oncol, 45, 884-91.

Kiberda BA, Keough-Ryana T, Clas CM (2003). Screening for prostate, breast and colorectal cancer in renal transplant recipients. Am J Trans, 3, 619-25.

Koleva-Kolarova RG, Zhan Z, Greuter MJ, et al (2015). Simulation models in population breast cancer screening: A systematic review. Breast $J, 24,354-63$.

Lee YC, Lin JT, Wu HM, et al (2007). Cost effectiveness analysis between primary and secondary preventive strategies for gastric cancer. Cancer Epidemiol Biomarkers Prev, 16, 875-85.

Leivo T, Salomaa A, Kosunen TU, et al (2004). Cost-benefit analysis of Helicobacter pylori screening. Health Policy, 70, 85-96.

Mason J, Axon AT, Forman D, et al (2002). The cost effectiveness of population Helicobacter pylori screening and treatment: a Markov model using economic data from a randomized controlled trial. Aliment Pharmacol Ther, 16, 559-68.

Ministry of Health, Labour and Welfare (2017). http://www. mhlw.go.jp/file/05-Shingikai-10901000-KenkoukyokuSoumuka/0000137850.pdf (accessed August 4, 2017).

Moher D, Liberati A, Tetzlaff J, et al (2009). Preferred reporting items for systematic reviews and meta-analyses: the PRISMA statement. Ann Intern Med, 151, 264-9.

Omidvari AH, Meester RG, Lansdorp-Vogelaar I (2016). Cost effectiveness of surveillance for GI cancers. Best Pract Res Clin Gastroenterol, 30, 879-91.

Owens DK, Whitlock EP, Henderson J, et al (2016). Use of decision models in the development of evidence-based clinical preventive services recommendations: Methods of the U.S. Preventive Services Task Force. Ann Intern Med, 165, 501-8.

Parsonnet J, Harris RA, Hack HM, et al (1996). Modelling cost effectiveness of Helicobacter pylori screening to prevent gastric cancer, a mandate for clinical trials. Lancet, 348, $150-4$.

Roderick P, Davies R, Raftery J, et al (2003). Cost effectiveness of population screening for Helicobacter pylori in preventing gastric cancer and peptic ulcer disease: using simulation. J Med Screen, 10, 148-56.

Shin DW, Yun YH, Choi IJ, et al (2009). Cost effectiveness of eradication of Helicobacter pylori in gastric cancer survivors after endoscopic resection of early gastric cancer. Helicobacter, 14, 536-44.

Terasawa T, Nishida H, Kato K, et al (2014). Prediction of gastric cancer development by serum pepsinogen test and Helicobacter pylori seropositivity in Eastern Asians: a systematic review and meta-analysis. PLoS One, 9, e109783.

Van Oortmarssen GJ, Boer R, Habbema JD (1995). Modelling issues in cancer screening. Stat Methods Med Res, 4, 33-54. 
Xie F, Luo N, Blackhouse G, et al (2008). Cost effectiveness analysis of Helicobacter pylori screening in prevention of gastric cancer in Chinese. Int J Technol Assess Health Care, 24, 87-95.

Xie F, Luo N, Lee HP (2008). Cost effectiveness analysis of population-based serology screening and (13) C-urea breath test for Helicobacter pylori to prevent gastric cancer:a Markov model. World J Gastroenterol, 14, 3021-7.

Xie F, O'Reilly D, Ferrusi IL, et al (2009). Illustrating economic evaluation of diagnostic technologies, comparing Helicobacter pylori screening strategies in prevention of gastric cancer in Canada. J Am Coll Radiol, 6, 317-23.

Yeh JM, Hur C, Kuntz KM, et al (2010). Cost effectiveness of treatment and endoscopic surveillance of precancerous lesions to prevent gastric cancer. Cancer, 116, 2941-53.

Yeh JM, Hur C, Ward Z, et al (2016). Gastric adenocarcinoma screening and prevention in the era of new biomarker and endoscopic technologies: a cost effectiveness analysis. Gut, 65, 563-74.

Yeh JM, Kuntz KM, Ezzati M, et al (2009). Exploring the cost effectiveness of Helicobacter pylori screening to prevent gastric cancer in China in anticipation of clinical trial results. Int J Cancer, 124, 157-66.

Zhou HJ, Dan YY, Naidoo N, et al (2013). A cost effectiveness analysis evaluating endoscopic surveillance for gastric cancer for populations with low to intermediate risk. PLoS One, 8, e83959.

This work is licensed under a Creative Commons AttributionNon Commercial 4.0 International License. 